\title{
MONIC POLYNOMIALS AND GENERATING IDEALS EFFICIENTLY
}

\author{
BUDH NASHIER
}

\begin{abstract}
If $I$ is an ideal containing a monic polynomial in $R[T]$ where $R$ is a semilocal ring, then $I$ and $I / I^{2}$ require the same minimal number of generators. An ideal containing a monic polynomial in a polynomial ring need not possess any minimal set of generators having a monic as a part of it.
\end{abstract}

1. Introduction. We are concerned with rings which are commutative and Noetherian with identity. By the dimension of a ring we mean the Krull dimension and we shall have occasion only to deal with rings of finite $\operatorname{dimension}$. Let $A$ be a ring and let $M$ be a finitely generated $A$-module. We define $\mu(M)$ to be the least number of elements in $M$ required to generate $M$ as an $A$-module. The conormal bundle of an ideal $I$ in a ring $A$ is the group $I / I^{2}$ viewed as an $A / I$-module. Many algebraic properties of this module are intertwined with those of the ideal $I$. For instance, the content of an easily verifiable result is that $\mu\left(I / I^{2}\right) \leqslant \mu(I) \leqslant \mu\left(I / I^{2}\right)$ +1 . To see when the lower inequality becomes an equality has been the theme of many papers in the literature; and this equality depends heavily on how the ideal $I$ sits inside $A$. In this article we embark on the following problem: Let $A=R[T]$ be a polynomial ring. Let $I$ be an ideal in $A$ such that $I$ contains a monic polynomial. Is it true that $\mu(I)=\mu\left(I / I^{2}\right)$ ?

In a lovely paper [4] Satyagopal Mandal has shown that if $\mu\left(I / I^{2}\right) \geqslant \operatorname{dim}(A / I)$ +2 and $I$ contains a monic polynomial then indeed $\mu(I)=\mu\left(I / I^{2}\right)$. While it is not difficult to obtain a positive answer to the question above in the case when $R$ is semilocal, we suspect of no situation where the desired equality between $\mu(I)$ and $\mu\left(I / I^{2}\right)$ will fail. We cite examples of ideals without monic polynomials for which the equality does not hold. One may be curious to know whether a monic polynomial should appear as a part of some minimal set of generators for $I$ given that $I$ does contain monic polynomials. In general, the answer turns out to be in the negative.

2. Cases when equality holds. Let us begin with a simple but useful lemma.

LEMMA 2.1. If an ideal $I$ in a ring $A$ is contained in all but finitely many maximal ideals of $A$, then $\mu(I)=\mu\left(I / I^{2}\right)$.

Proof. Let $\mu\left(I / I^{2}\right)=n$. Choose elements $a_{1}, \ldots, a_{n}$ in $I$ which generate $I$ mod $I^{2}$. If $I$ is contained in all the maximal ideals of $A$, then $\left(a_{1}, \ldots, a_{n}\right)=I$. Otherwise, let

Received by the editors December 17, 1984.

1980 Mathematics Subject Classification. Primary 13F20. 
$M_{1}, \ldots, M_{r}$ be all those maximal ideals of $A$ that do not contain $I$. We can rearrange these $M_{i}$ 's to assume that $a_{1}$ is in $M_{1}, \ldots, M_{t}$ but not in $M_{t+1}, \ldots, M_{r}$. Then the ideal $J=I^{2} \cap M_{t+1} \cap \cdots \cap M_{r}$ is not contained in $M_{1} \cup M_{2} \cup \cdots \cup M_{t}$. We can find an element $b$ in $J$ such that $b$ is outside $M_{1} \cup M_{2} \cup \cdots \cup M_{t}$. Replace $a_{1}$ by $a_{1}+b=a$. Then $a, a_{2}, \ldots, a_{n}$ generate $I$ since they do so locally.

Corollary 2.2. Let $A$ be a semilocal ring. Then $\mu(I)=\mu\left(I / I^{2}\right)$ for any ideal $I$ in A.

As a consequence we obtain

TheOREM 2.3. Let $A=R[X]$ where $R$ is a semilocal ring. Let $I$ be an ideal in $A$ such that I contains a monic polynomial. Then $\mu(I)=\mu\left(I / I^{2}\right)$. Further, we can find a minimal set of generators for I such that one (hence all) elements in this set are monic polynomials.

Proof. Let $\mu\left(I / I^{2}\right)=n$. Let $a$ be a part of a minimal set of generators for $I$ mod $I^{2}$. Let $f$ be a monic polynomial in $I$. Replace $a$ by $a+f^{n}$ for suitable $n$ to assume that $a$ is monic. Then $I_{1}=I /(a)$ is an ideal in $A_{1}=A /(a)$ and $\mu\left(I_{1} / I_{1}^{2}\right)=$ $n-1$. Now $A_{1}$ is semilocal as it is integral over $R$. By Corollary $2.2 \mu\left(I_{1}\right)=n-1$. Hence, $\mu(I)=n$. Further, since $a$ appears as a part of a minimal set of generators for $I$ we can add powers of $a$ to the other generators to obtain that each one of them is monic.

COROLlaRY 2.4. If $R$ is a semilocal ring and if $I$ is an ideal containing a monic polynomial in $R[X]$ such that projective dimension of $I$ is finite and $I / I^{2}$ is a free $R[X] / I$-module, then $I$ is generated by a regular sequence.

PROof. By Ferrand [1] or Vasconcelos [5] the grade of $I$ equals $\operatorname{rank}\left(I / I^{2}\right)$. By Theorem $2.3, \mu(I)=\mu\left(I / I^{2}\right)=$ grade of $I$, therefore $I$ is generated by a regular sequence [see $2,11.11$ ].

The following example shows that Theorem 2.3 does not extend to ideals that do not contain a monic polynomial.

EXAMPLE 2.5. Let $R=k\left[\left[t^{2}, t^{3}\right]\right]$. Let $M$ be the ideal in $R[X]$ generated by $t^{2}-t^{3} X$ and $1-t^{2} X^{2}$. One easily verifies that $M$ is a maximal ideal of height 1 in $R[X]$. Since $M \cap R=(0), \mu\left(M / M^{2}\right)=1$. But $M$ cannot be generated by a single element as can be seen without much ado.

The above example involves an element of $\operatorname{Pic}(R[X])$ which is not extended from $R$.

EXAmple 2.6. Let $D=\mathbf{R}[X, Y] /\left(X^{2}+Y^{2}-1\right)=\mathbf{R}[x, y]$. Then $D$ is a Dedekind domain. Consider the ideal $I$ generated by $(1+y) T-x$ and $x T-1+y$ in $D[T]$. Then $I$ is in $\operatorname{Pic}(D[T])$ such that $\mu\left(I / I^{2}\right)=1$ and $\mu(I)=2$.

Proof. Let us first see that $I / I^{2}$ is a free $D[T] / I$-module of rank 1 . For this, we observe that $(I, 1+y)=D[T]$. Hence $1+y$ becomes a unit in $D[T] / I$. Then it is easy to see that $I \bmod I^{2}$ is generated by the image of $(1+y) T-x$. Now $\mu\left(I / I^{2}\right)$ $=1$ implies that $I$ is a projective ideal of rank one and hence an element of 
Pic $(D[T])$. Suppose that $I$ is principal. Whence it follows that the constant terms of the elements of $I$ generate a principal ideal. But the ideal generated by the constant terms of elements of $I$ is the maximal ideal $(x, y-1)$ which, being a real point on $S^{1}$, requires two generators. Therefore, $\mu(I)=2$. Furthermore, $I$ as an element of $\operatorname{Pic}(D[T])$ is extended from $R$ since $D$ is normal.

The following remark shows that neither of the ideals in the examples above contains a monic polynomial.

Remark 2.7. Let $A=R[T]$ be a polynomial ring. Let $I$ be an ideal containing a monic polynomial in $A$. If $\mu\left(I / I^{2}\right)=1$ then $\mu(I)=1$.

Proof. The given hypothesis implies that $I$ is a projective ideal of rank 1 . Since $I$ contains a monic polynomial, by a theorem of Quillen and Suslin (see [3]), I must be principal.

While the presence of a monic polynomial in an ideal $I$ plays such an important role in determining the cardinality of a minimal set of generators for $I$, one may ask the following: Suppose that an ideal $I$ in a polynomial ring $R[T]$ contains a monic polynomial and $\mu(I)=n$. Is it possible to find a set of $n$ generators for $I$ such that one of them is monic? Curiously enough, the following example illustrates that the answer is no.

EXAMPLE 2.8. Take a Dedekind domain $D$ whose class group has elements of infinite order. To wit, the coordinate ring of the smooth elliptic curve: $Y^{2}+Y=X^{3}$ - $X$. Choose a prime $P$ in $D$ of infinite order. Then $(P, T)=M$ is a maximal ideal in $D[T]$ such that $\mu(M)=2[2,16.1]$. We claim that $M$ cannot be generated by two polynomials such that one of them is monic. Suppose, if possible, that $M$ is generated by $f$ and $g$ in $D[T]$ and that $f$ is monic.

It is a well-known fact that the ideal generated in $D$ by the resultant of $f$ and $g$ is primary to $P$. Hence $P$ should have finite order-contradiction.

It is a pleasure for me to thank Warren Nichols for steady and useful conversations.

\section{REFERENCES}

1. D. Ferrand, Suites régulière et intersection complète, C. R. Acad. Sci. Paris Ser. A 264 (1967), A427-A428.

2. A. V. Geramita and C. Small, Introduction to homological methods in commutative rings, Queen's Papers in Pure and Applied Mathematics, Vol. 43 (2nd ed.), Kingston, Ontario, Canada, 1979.

3. T. Y. Lam, Serre's conjecture, Lecture Notes in Math., No. 635, Springer-Verlag, Berlin and Heidelberg, 1978.

4. S. Mandal, On efficient generation of ideals, Invent. Math. 75 (1984), 59-67.

5. W. Vasconcelos, Ideals generated by R-sequences, J. Algebra 6 (1967), 309-316.

Department of Mathematics, Florida State University, Tallahassee, Florida 32306 